\title{
Variability in the Last Abdominal Sternum of Brontispa longissima Populations Using Outline-Based Geometric Morphometric Analysis
}

\author{
Abigail R. Cuyacot, Emma M. Sabado, and Cesar G. Demayo
}

\begin{abstract}
The coconut plant plays a major role in the economy of many Asian countries including the Philippines and their economies are recently threatened due to a serious outbreak of the coconut leaf beetle, Brontispa longissima (Gestro). This study was conducted to observe if there is an intraspecific morphological variation among the coconut hispine beetle populations in terms of the last abdominal sternum shape to better understand why these pests differed in the level of their infestation in different populations. From outline-based geometric morphometrics analysis, results showed that a considerable sternum shape variation among the populations is studied. Morphological sternum shape variations were verified statistically in Principal Component Analysis, Canonical Variate Analysis, and Multivariate Analysis of Variance (MANOVA) using PAST 2.13 software. Furthermore, shape differences could be observed in the shape representation of Elliptic Fourier Shape analysis as well as in the PCA diagrams and CVA scatter plots.
\end{abstract}

Index Terms-Abdominal sternum, B. longissima, geometric morphometircs, morphology, shape variability.

\section{INTRODUCTION}

The coconut palm plays a major role in the economy of many Asian countries by providing food and income from its products. It is an important source of income in many households especially in the Philippines which is known to be the biggest exporter of coconut productions in the world. Currently, there are losses of coconut production that lead to a threatened economy of the country due to a serious outbreak of the coconut hispine beetle, Brontispa longissima (Gestro), (Coleoptera: Chrysomelodae) [1]. The larvae and adult of the coconut hispine beetles (CHB) feed on tissues of unopened leaf buds of the palm where they turn the leaves brown causing a decreased fruit production and successive severe defoliations that leads to death of the tree [2]. Only limited information about $B$. longissima has been presently published Yet there have been available reports associating the management of this pest. Yet, the reported control strategies which seem to have failed to the infestation are now distributed in the Southern places of the Philippines, in

Manuscript received February 23, 2014; revised April 28, 2014. This work was supported in part by the Department of Science and Technology-ASTHRDP.

Abigail R. Cuyacot and Cesar G. Demayo are with the Department of Biological Sciences, CSM, MSU-IIT, Iligan City, Philippines (e-mail: abigailcuyacot@gmail.com).

Emma M. Sabado is with the Plant Science Department, COA, MSU-Main Campus, Marawi City, Philippines (e-mail: emcsab@yahoo.com).
Mindanao.

Moreover, it is argued that a successful control of the pest based on correct identification for the inability to recognize distinct populations can have severe and costly consequences for pest management [3]. Therefore, it's necessary to consider the coconut hispine beetle (CHB) in details to stop its infestation in the country, especially that different levels of infestation have been observed among the invaded localities.

Morphological variability is a good start to better understand why the CHB have differed in the level of infestation, for distinct populations of agronomic pest differ only on little phenotypic characters and sometimes these may represent stable characters with genetic bases which are good bases for the study [4]. An advanced tools of the geometric morphometrics (GM) is applied in this study described morphological diversity of $B$. longissima from different locations. Geometric morphometrics (GM) have a advantage to provide a consistent set of shape variables for hypothesis testing [5] and deliver the sounds graphic analyses that quantify and visualize morphometric variation within and between the organisms [6]. This study uses outline-based GM analysis to extract the margin around the specimen and this is conducted to gain information on intraspecific variation of both female and male $B$. longissima that might explain its fitness. Since the last abdominal sternum of beetles is assosiated with a chitinous apodeme of variable shapes, [7] this study is therefore conducted in this structure to determine patterns of variation among populations of the coconut hispine beetle, $B$. longissima.

\section{MATERIALS AND METHODS}

\section{A. Collection and Preparation of Samples}

Adult coconut leaf beetles were collected from different infested places in Mindanao, Philippines namely; (a.) Aloran, Misamis Occidental, (b.) Aurora, Zamboanga del Sur, (c.) Marawi City, Lanao del Sur (d.) Pagadian City, Zamboanga del Sur (e.) Pantao Ragat, Lanao del Norte, (f.) Parang, Maguindanao, and (g.) Wao, Lanao del Sur. The collection was done manually by picking up the coconut beetles. The collected insects were placed inside by glasses containing prepared fixative (70\% ethyl alcohol $+30 \%$ glacial acetic acid) for preservation. The images of the last abdominal sternum (Fig. 1) were captured by using the built-in digital camera of Olympus SZ60 Stereomicroscope. A total of 60 specimens (30 males and 30 females) per location were 
prepared in the study for the outline-based geometric morphometric analysis.

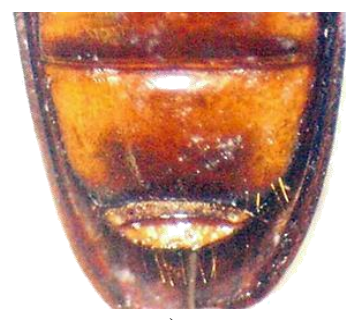

a) b)

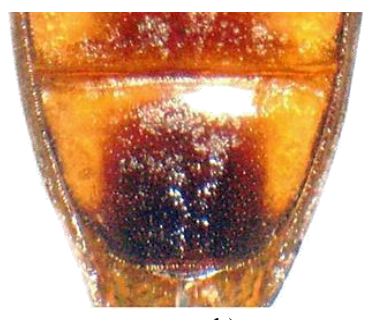

Fig. 1. Photograph of the last abdominal sternum of coconut leaf beetle; a) male and b) female.

\section{B. Acquisition of Outline Data}

Outline-based geometric morphometric analysis which extracts the margin around the specimen was used to analyse the shape of the last abdominal sternum of the coconut leaf beetles by a digitizing program tpsDig ver. 2.12 software [8] where it allowed saving the data into a thin plate spline (TPS) file. A total of 100 closely connected points along the shape of the last abdominal sternum in the images of the specimen were established (Fig. 2). The tps curve was converted into the landmark points (XY coordinates) to serve as raw data for outline analysis. The information of the non-shape data was held to be constant to remove the non-shape variation [3].

All specimens were digitized with three replicates in order to reduce the measurement error [9] and these were aligned by using a Generalized Procrustes Analysis (GPA) to superimpose the landmark configurations with least-squares estimates for translation and rotation parameters [10], thus they removed the non-shape effects of translation, rotation, and scale [11]. Each set of analysis under consideration was conducted separately for males and females.

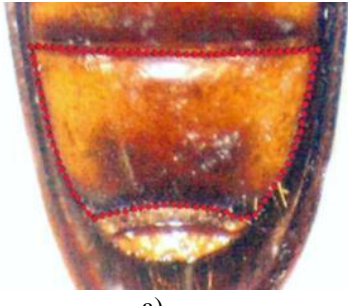

a)

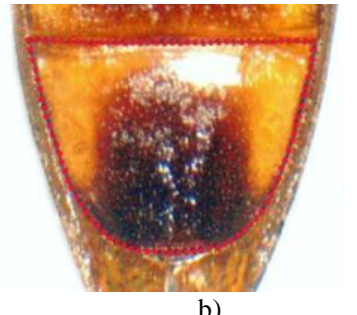

b)
Fig. 2. Coordinates of the outlines of the morphological structure of coconut leaf beetle's last abdominal sternum (a. male and b. female).

\section{Data Analysis}

Thin-plate splines (TPS) were used to graphically illustrate the patterns of shape variations established by the outline-based geometric morphometric analysis which represented the transformation of the reference to each specimen [12]. The raw XY coordinates were first superimposed using a generalized procrustes method, where the sum of squared distances between each object and a reference configuration were iteratively minimized by translation and rigid rotation [13]. The superimposed coordinates of the female and the male populations of $\mathrm{CHB}$ were subjected to standard method of outline analysis, the Elliptic Fourier Shapes (EFS) using the algorithm in PAST [14]. The coordinates of superimposed configurations in all aligned specimens were also used for Principal Component Analysis (PCA) and Canonical Variate Analysis (CVA) to explore patterns of variation in the last abdominal sternum among the female and the male CHB populations and to analyze and test the differences [15]. Data were also subjected to multivariate analysis of variance (MANOVA) using PAST software version 2.15 to determine whether the last abdominal sternum shape differed significantly from populations [16]. Kruskal-Wallis Test was also made to the significant PC scores to compare with the population mean ranks [17] whether they were taken from a population with equal medians. PCA scatter diagram, CVA scatter plot and XY graph were made in order to compare patterns of intraspecific variation.

\section{RESULTS AND DISCUSSION}

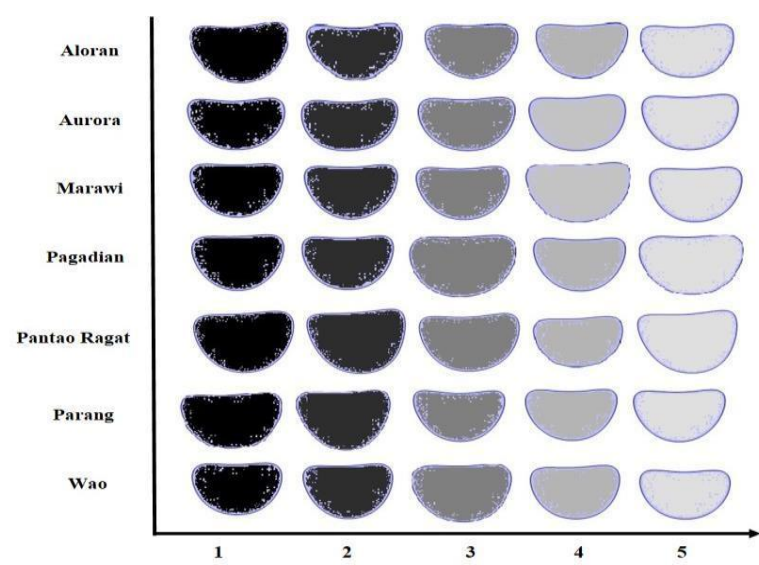

Fig. 3. Modes of shape change represented by elliptic fourier shapes (EFS) of female Brontispa longissima populations; dark to light shading specifies representative shapes from EFS 1 to 5 of 3 modes.

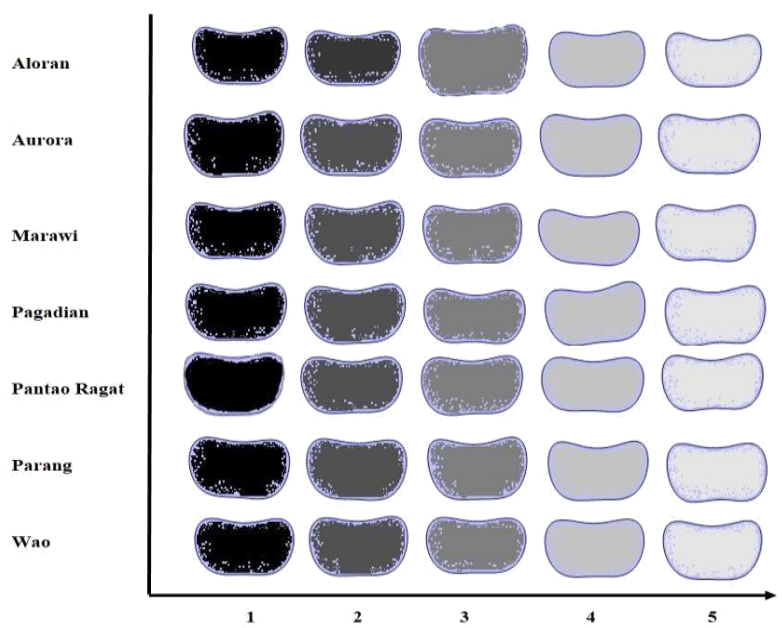

Fig. 4. Modes of shape change represented by elliptic fourier shapes (EFS) of male Brontispa longissima populations; dark to light shading specifies representative shapes from EFS 1 to 5 of 3 modes.

Elliptic fourier shapes (EFS) were produced with three modes via standard analysis (elliptic fourier 2D of PAST 2.15 software) of the CHB last abdominal sternum digitized outlines (Fig. 3 and Fig. 4). Sternite shapes from different populations could be observed by the trends in shape variation captured by each elliptic fourier shapes (only up to EFS 5 was chosen for representation). It is possible to describe corresponding biological change seen in the extant 
CHB datasets. Almost all of the shapes exhibited a considerable difference in their lateral and longitudinal lengths which corresponded to each of the locations of the female and male beetles. We observed that some had wide orientation while others had the most pointed sternite shapes. A Multivariate Analysis of Variance (MANOVA) was carried out to see whether any morphological differences were detected among the populations of the female and the male $B$. longissima in their last abdominal sternum had in this case, the analysis resulted in the populations having significant morphological variation (Fig. 7 and Fig. 8). The PCA scatter diagram was also made, as shown in Fig. 5, to support the findings. The diagrams show variation in sternum shape of $\mathrm{CHB}$ populations having $\mathrm{PC} 1=55.83 \%, \mathrm{PC} 2=14.63 \%$ and $\mathrm{PC} 3=9.08 \%$ as the highest $\%$ variance in females and $\mathrm{PC} 1=52.16 \%, \mathrm{PC} 2=15.88 \%$ and $\mathrm{PC} 3=12.62$ in males.

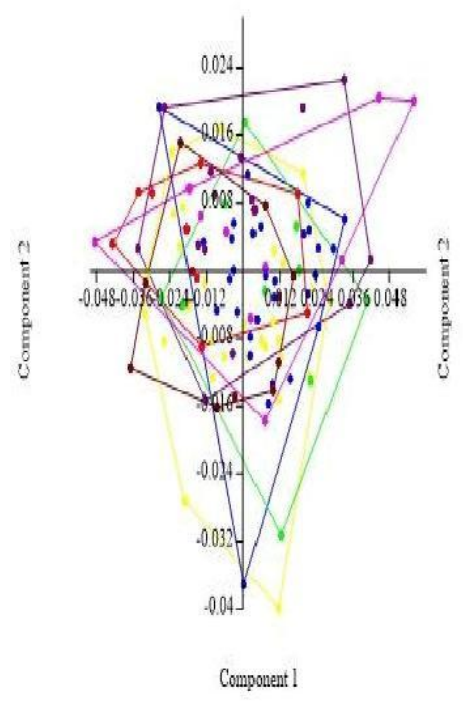

a)

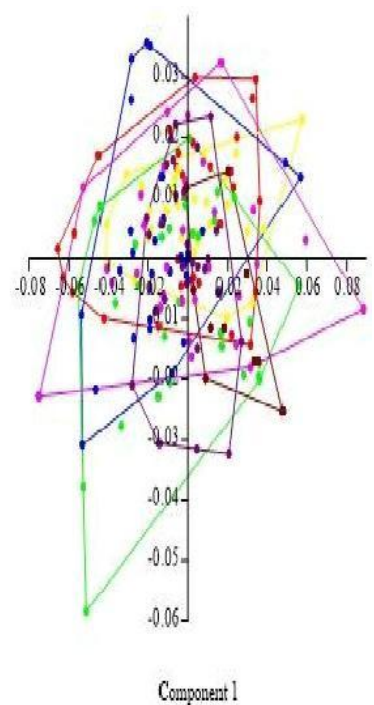

b)
Aloran Aurora Marawi Pagadian Pantao Ragat 1 Parang Fig. 5. Principal component scatter diagram of female a) and male b) last abdominal sternum of Brontispa longissima populations.

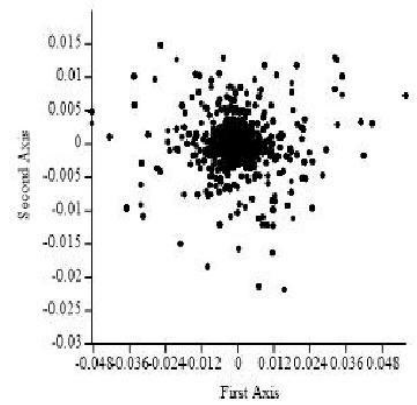

a)

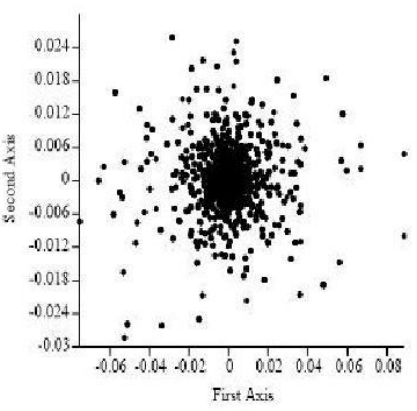

b)
Fig. 6. The top axes of variations (PC1 and PC2) of the last abdominal sternum of coconut hispine beetle population (a.female and b.male).

The possible factors that affect these variations might be due to the pheromone production, mating behaviour, egg production and release, because these are all handled largely by the abdomen and particularly by modifications of the last abdominal segments (sternum-ventral abdominal segment) [18]. It implies that the reproductive structures are located on the last abdominal segments and in B. longissima, the last abdominal sternum is the 6th (in male) and 5th in female which are the structures conducted in this study. Fig. 6 displays the top two axes of variations based on the principal component scores from the last abdominal sternum shape of coconut hispine beetle populations; variabilities that redirect toward the center of the axis and the others being widely dispersed. Moreover, we hypothesized that the first axis reflects genetic variation and the other manifests the environmental factors that may affect sternum shapes. These morphological disparities on the sternum shape could be due to the isolation that affects the populations studied where the gene flow could be interrupted between them in which the $B$. longissima group will show a particularly high plasticity in the capacity to withstand differences and environmental pressures enacted in each particular environment [19]. This capacity could then be reflected in high morphological plasticity that emerges and indicates that the populations are evolving [20]. In the case of CHB sternum shapes, the last segments, like most of the beetles, are the genital segments that retain ancestral appendages that have been modified into the genitalia of the male and female, that is, for holding the female in position during copulation (male) and in the female accepting the male during copulation and also for oviposition or egg laying [21]. In addition, many studies have documented the influence of sexual selection in shaping and diversifying morphological, physiological, and behavioral traits of both sexes [22]. Sexual selection arises because individuals vary in reproductive success [23] and it is often identified to be a powerful evolutionary force, because variation in reproductive success often exceeds that of other fitness components which include survivorship [24]. The results presented here indicate that morphological variations were mainly due to differences in the last abdominal sternum shape and this may corroborate the frequently suggested report that morphological variation of individuals may be strongly dependent upon unfavorable environmental conditions [25].

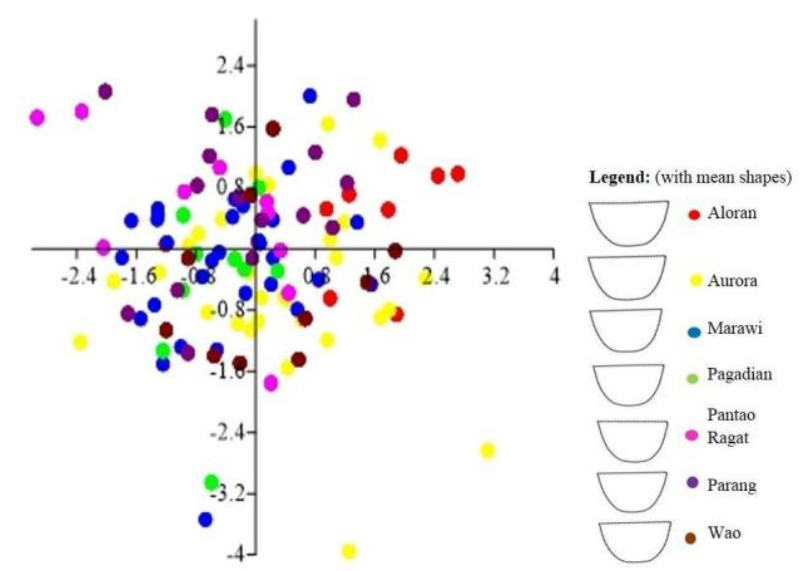

Fig. 7. CVA scatter plot showing the female distribution of last sternum shapes from different locations of coconut leaf beetle samples based on outline geometric morphometric analysis. Results of MANOVA test for significant variation in the last abdominal sternum shape: Wilks' lambda $=0.6024$, df $1=18$, df $2=1757, F=19.15$ and $p($ same $)=2.216^{-56}$.

From the significant variation of the last abdominal sternum of $\mathrm{CHB}$ populations viewed from MANOVA test results (Fig. 7 and Fig. 8), data has been further verified by non-parametric Kruskal-Wallis to test whether samples originate from the same distribution (Table I and Table II). The statistics generated significant outcome for female ( $p$ $=8.795-11<0.05)$ and male $(p=2.924-11<0.05$. Thus, there were significant differences between the medians of at least two populations. 


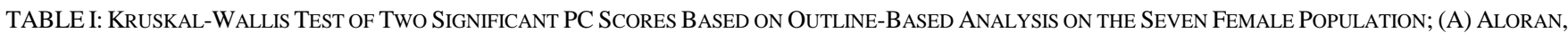
(B) Aurora, (C) Marawi, (D) Pagadian, (E) Patao Ragat, (F) PARANG AND (G) WaO. Bold Numbers Indicate Significant DifFERENCE (a=0.05)

\begin{tabular}{|c|c|c|c|c|c|c|c|}
\hline & A & $\mathrm{B}$ & $\mathrm{C}$ & $\mathrm{D}$ & $\mathrm{E}$ & $\mathrm{F}$ & $\mathrm{G}$ \\
\hline & & & $5.62 \mathrm{E}-$ & 0.0004 & $1.98 \mathrm{E}-$ & 6.52E- & \\
\hline \multirow[t]{2}{*}{ A } & & 0.3817 & 06 & 18 & 05 & 05 & 0.7431 \\
\hline & & & 6.42E- & 0.0004 & 9.40E- & 0.0002 & \\
\hline B & 0.0001 & 0.0001 & & & & & 9.40E- \\
\hline \multirow[t]{2}{*}{$\mathrm{C}$} & 18 & 35 & & 0.444 & 0.3386 & 0.8272 & 06 \\
\hline & 0.0087 & 0.0087 & & & & & 0.00022 \\
\hline \multirow[t]{2}{*}{ D } & 76 & 93 & 1 & & 0.1008 & 0.3526 & 2 \\
\hline & 0.0004 & 0.0001 & & & & & 4.08E- \\
\hline \multirow[t]{2}{*}{$\mathrm{E}$} & 16 & 97 & 1 & 1 & & 0.5118 & 05 \\
\hline & 0.0013 & 0.0052 & & & & & 4.09E- \\
\hline \multirow[t]{2}{*}{$\mathrm{F}$} & 69 & 05 & 1 & 1 & 1 & & 05 \\
\hline & & & 0.00019 & 0.0046 & 0.00085 & 0.0008 & \\
\hline $\mathrm{G}$ & 1 & 1 & 7 & 61 & 7 & 58 & 0 \\
\hline
\end{tabular}

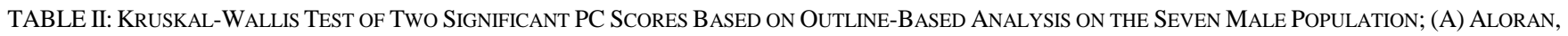

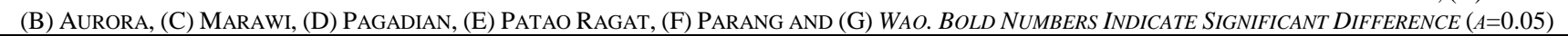

\begin{tabular}{|c|c|c|c|c|c|c|c|}
\hline & $\mathrm{A}$ & B & $\mathrm{C}$ & $\mathrm{D}$ & $E$ & $\mathrm{~F}$ & $\mathrm{G}$ \\
\hline & & & 3.19E- & 5.39E- & & 0.002 & \\
\hline \multirow[t]{2}{*}{ A } & & 0.8916 & 06 & 05 & 0.0568 & 711 & 0.7848 \\
\hline & & & 3.30E- & 8.66E- & 0.0169 & 0.000 & \\
\hline B & $6.71 \mathrm{E}$ & 6.93E- & & & 0.0016 & 0.002 & 3.15E- \\
\hline \multirow[t]{2}{*}{$\mathrm{C}$} & -05 & 08 & & 0.5661 & 12 & 477 & 07 \\
\hline & 0.001 & 0.00018 & & & & 0.043 & $6.40 \mathrm{E}-$ \\
\hline \multirow[t]{2}{*}{$\mathrm{D}$} & 132 & 2 & 1 & & 0.0178 & 04 & 06 \\
\hline & & & & & & 0.517 & \\
\hline \multirow[t]{2}{*}{$\mathrm{E}$} & 1 & 0.3558 & 0.03385 & 0.3738 & & 8 & 0.02869 \\
\hline & 0.056 & 0.00621 & & & & & 0.00199 \\
\hline \multirow[t]{2}{*}{$\mathrm{F}$} & 93 & 8 & 0.05202 & 0.9038 & 1 & & 9 \\
\hline & & & 6.61E- & 0.00013 & & 0.041 & \\
\hline $\mathrm{G}$ & 1 & 1 & 06 & 4 & 0.6025 & 98 & 0 \\
\hline
\end{tabular}

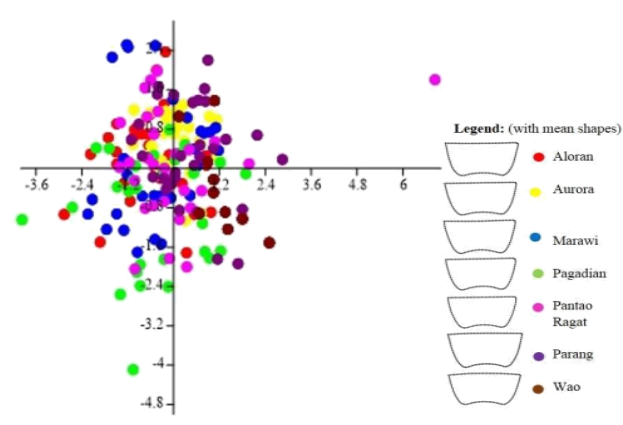

Fig. 8. CVA scatter plot showing the male distribution of last sternum shapes from different locations of coconut leaf beetle samples based on outline geometric morphometric analysis. Results of MANOVA test for significant variation in the last abdominal sternum shape: Wilks' lambda $=\mathbf{0 . 5 8 0 6}$, df $1=18$, df $2=1757, F=20.69$ and $p($ same $)=3.767^{-61}$.

Morphological differences in CHB populations were found in this study which resulted consistently in all of the analyses made. Findings of outline-based geometric morphometric analysis have shown the last abdominal sternum shape variation among the female and the male CHB populations. It can be observed in the results obtained that outline-based analysis is an enhanced means of discerning intraspecific morphological variation aside from the fact that it is a precise and specific method. It further confirmed the effectiveness of geometric morphometric techniques in describing morphological variation in an organism between populations.

\section{CONCLUSION}

The results have shown that $B$. longissima (coconut hispine beetle) populations significantly differed in terms of the last abdominal sternum shapes. The reasons for intraspecific variation on sternum of $\mathrm{CHB}$ and the degree to which they represent are still indefinite and more further studies are needed. The shape of diverse morphological structures reflects their function in nature, thus, further understanding on the shape and structure as well as the function of the last abdominal sternum of CHB would lead to the developmental genetics of the insect.

\section{ACKNOWLEDGMENT}

The researchers would like to thank the Department of Science and Technology-Accelerated Science and Technology Human Resource Development Program (DOST-ASTHRDP) for funding this study, the Department of Biological Sciences of MSU-IIT for making this research possible and Mr. Muhmin Michael E. Manting for the technical assistance.

\section{REFERENCES}

[1] H. T. T. Giang and S. Nakamura, "The Study on Biological Characteristics of Brontispa longissima (GESTRO) (COLEOPTERA: CHRYSOMELIDAE)," Japan J. Sci. Dev., vol. 7, pp. 159-164, 2009.

[2] S. Nakamura, K. Konishi, and K. Takasu, "Invasion of the coconut hispine beetle, Brontispa longissima: Current situation and control measures in Southeast Asia," in Proc. international workshop on development of database (APASD) for biological invasion, vol. 3, pp. $1-9,2006$.

[3] L. M. L. D. Cruz, M. A. J. Torres, A. T. Barrion, R. Joshi, L. S. Sebastian, and C. G. Demayo, "Geometric morphometric analysis of the head, pronotum and genitalia of the rice black bug associates with selected rice types," Egyptian Academic Journal of Biological Sciences, vol. 4, no. 1, pp. 21-31, 2011.

[4] M. A. J. Torres, J. Lumansoc, and C. G. Demayo, "Variability in head shapes in three populations of the Rice Bug Leptocorisa oratorius (Fabricius) (Hemiptera: Alydidae)," Egypt. Acad. J. biolog. Sci., vol. 3, no. 1, pp. 173-184, 2010. 
[5] D. C. Adams, "Methods for shape analysis of landmark data from articulated structures," Evolutionary Ecology Research, vol. 1, pp. 959-970, 1999.

[6] H. A. Benitez, "Sexual Dimorphism Using Geometric Morphometric Approach," 2012, doi 10.5772/55195.

[7] M. Erbey, S. Candan, and E. Kocak, "External Morphology of the Female Genitalia of Lixus nordmanni Hochhuth, 1847 (Coleoptera: Curculionidae, Lixinae): A Scanning Electron Microscope Study," GU $J$ Sci, vol. 23, no. 4, pp. 385-391, 2010.

[8] F. J. Rohlf, "tpsDig-Thin Plate Spline Digitizer Version 2.0," Department of Ecology and Evolution, State University of New York at Stony Brook, New York, 2004.

[9] V. Dvorak, A. M. Aytekin, B. Alten, S. Skarupova, J. Votypka, and P. Volf, "A comparison of the intraspecific variability of Phlebotomus sergenti Parrot, 1917 (Diptera: Psychodidae)," Journal Vector Ecology, vol. 31, no. 2, pp. 229-238, 2005.

[10] D. C. Adams, F. J. Rohlf, and D. E. Slice, "Geometric morphometrics: ten years of progress following the 'revolution'," Italian Journal of Zoology, vol. 71, pp. 5-16, 2004.

[11] A. Pizzo, A. Roggero, C. Palestrini, A. P. Moczek, and A. Rolando, "Rapid shape divergences between natural and introduced populations of a horned beetle partly mirror divergences between species," Evolution and Develop, vol. 10, no. 2, pp. 166-175, 2008.

[12] F. L. Bookstein, Morphometric tools for landmark data: Geometry and Biology, New York: Cambridge University Press, pp. 435, 1991.

[13] F. J. Rohlf, "Rotational fit (Procrustes) methods," in Proc. the Michigan Morphometric Workshop, F. J. Rohlf and F. L. Bookstein eds., Ann Arbor: University of Michigan Museum of Zoology, no. 2, pp. 227-236, 1990.

[14] S. F. Ferson, F. J. Rohlf, and R. K. Koehn, "Measuring shape variation of two-dimensional outlines," Systematic Zoology, vol. 34, pp. 59-68, 1985.

[15] R. Dhivya and K. Manimegalai, "Wing Shape Analysis of the Japanese encephalitis vector Culex gelidus (Diptera: Culicidae) at the Foot Hill of Southern Western Ghats, India," World Journal of Zoology, vol. 8, no. 1, pp. 119-125, 2013.

[16] O. Hammer, D. A. T. Harper, and P. D. Ryan, "PAST: Paleontological Statistics Software Package for Education and Data Analysis," Palaeontologia Electronica, vol. 4, no. 1, p. 9, 2001.

[17] V. Bewick, L. Cheek, and J. Ball, "Statistics review 10: Further nonparametric methods," Critical Care, vol. 8, pp. 196-199, 2004.

[18] B. K. Mitchell and J. S. Scott, "An Introduction to Insect Structure. DBS, University of Alberta," Supported in part by Academic Technologies for Learning and Faculty of Science, University of Alberta.

[19] M. Palmer, "Testing the 'island rule' for a tenebrionid beetle (Coleoptera, Tenebrionidae)," Acta Oecologica-International Journal of Ecology, vol. 23, pp. 103-107, 2002.
[20] H. A. Benítez, J. Pizarro-Araya, R. Bravi, M. J. Sanzana and F. M. Alfaro, "Morphological variation on isolated populations of Praocis (Praocis) spinolai," Journal of Insect Science, vol. 14, no. 11, 2014.

[21] J. G. Houseman. Insect External Anatomy. [Online]. Available: http://salinella.bio.uottawa.ca/BIO3323/Labs/PDFs/BIO3323Lab02_ ExternalAnat_04.pdf

[22] T. R. Birkhead, D. J. Hosken, and S. Pitnick, Sperm Biology: An Evolutionary Perspective, San Diego USA: Academic Press, 2009.

[23] M. Demont, "Polyandry and postcopulatory sexual selection in yellow dung flies," Zurich Open Repository and Archive, University of Zurich, Faculty of Science, 2010.

[24] G. Arnqvist and M. Kirkpatrick, "The evolution of infidelity in socially monogamous passerines: The strength of direct and indirect selection on extrapair copulation behavior in females," American Naturalist, vol. 165 , pp. 26-37, 2005.

[25] H. Tatsuta, K. Mizota, and S. I. Akimoto, "Allometric patterns of heads and genitalia in the stag beetle Lucanus maculifemoratus (Coleoptera: Lucanidae)," Annals of the Entomological Society of America, vol. 94, pp. 462-466, 2001.

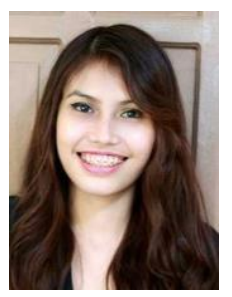

Abigail R. Cuyacot is currently pursuing her degree of master of science in biology at Mindanao State University-Iligan Institute of Technology, A. Bonifacio Avenue, Iligan City, Philippines.

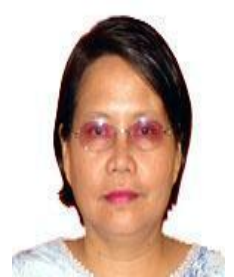

Emma M. Sabado is currently the professor of Plant Science Department, College of Agriculture, Mindanao State University-Main Campus, Marawi City, Philippines. Her expertise includes entomology and plant pathology/Ag-education. She is the in-charge of the Butterfly Garden \& Strawberry Production Project of the College of Agriculture.

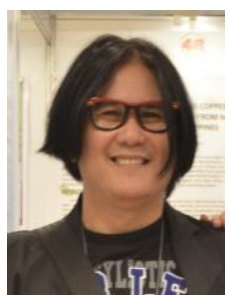

Cesar G. Demayo is currently the chairman and professor of the Department of Biological Sciences, College of Science and Mathematics, MSU-Iligan Institute of Technology, Iligan City, Philippines. His researches include environmental toxicology, biodiversity and genetics. He is an active member of the Philippine Society for the Study of Nature and the Pest Management Council of the Philippines. 